\title{
INFINITE UNITARY GROUPS
}

\author{
BY \\ RICHARD V. KADISON( $\left.{ }^{1}\right)$
}

1. Introduction. The investigation carried out in this paper finds its origin in $[2]\left({ }^{2}\right)$. While examining the possibility of constructing a "signed" determinant theory in a factor $\left(^{3}\right)$ of type $\mathrm{II}_{1}$, it was proved in [2] that there exists no nontrivial uniformly continuous character on the group of unitary operators in a factor not of type $\mathrm{I}_{n}$ ( $n$ finite). This raises the question of the nature of the representations of the unitary groups of the various factors and, indeed, the entire question of the structure theory of these groups. The detailed study of the classical groups has yielded a beautiful and useful mathematical regime; there is every reason to believe that the theory of the infinite-dimensional analogues of these groups will share, to a certain extent, the beauty and usefulness of the classical theory. One might wonder whether or not such a study was appropriate at this stage of mathematical development. When viewed as topological groups, in the uniform (operator bound) topology, these groups are not locally compact, and we are far from being able to answer the natural questions about non-abelian locally compact groups. Although their topology alone does not bring these groups within the scope of such powerful tools as invariant integration, these groups have special features which make them amenable to detailed investigation. One has, for example, the maneuverability afforded by the underlying Hilbert space and by the knowledge that the given group is the full set of unitaries of some factor. This last fact alone puts at our command the methods of $[5,6]$, and, at least in the case of the groups of factors of type $\mathrm{II}_{1}$, makes available to us the trace and determinant theory.

It seems more than likely that, in the case of a factor of type $\mathrm{II}_{1}$, it may eventually be possible to study the "neo-classical groups" in a manner similar to that in which one deals with a Lie group and its Lie algebra. Indeed, our results indicate a striking relation between the ideal-theoretic structure of a factor (as determined in [3]) and the invariant subgroup structure of its unitary group (the ideal-theoretic structure of the factor undoubtedly reflects itself in the Lie ideal structure of any natural Lie algebra one would attach to the unitary group, e.g., in the $\mathrm{II}_{1}$ case, the skew-adjoint operators

Presented to the Society, October 27, 1951; received by the editors August 15, 1951.

(1) The author is a National Research Fellow.

(2) Numbers in brackets refer to the bibliography at the end of this paper.

${ }^{(3)}$ We shall make free use of the general results and techniques of $[5,6]$ without further reference. When particular facts in these papers are needed, "e shall reler to the paper: by: the abbreviation R O. 1 and 11 
belonging to the factor). It is not in this general spirit, however, that we have conducted our examination of the closed invariant subgroups of the unitary group of a factor; nor have we done anything to carry the program indicated by the preceding remarks past this examination. We have, rather, taken full advantage of the special devices which this special situation provides. The particular results may ultimately point the way to the general theory.

We deal only with the various unitary groups in this paper and not, as might seem natural and appropriate, with the various general linear groups (i.e., the full set of invertible operators in a factor). The question of the infinite-dimensional general linear groups will be treated in a subsequent publication. The investigation of the general linear groups leans heavily upon the results of [2] whereas the study of the unitary groups does not require these results (although it is motivated and directed by them). It is for this reason that we present the two sets of results separately.

In $\$ 2$ we collect various results needed to prove the principal theorems. This section consists primarily of arguments in lemma form which would appear again and again in the individual investigations of the unitary groups of the various types of factors. $\$ 3$ contains the statements and proofs of the main results. It is proved in this section that the only closed normal subgroups of the unitary group of a factor of type $\mathrm{II}_{1}$ or III are the closed normal subgroups of the center (cf. Theorem 2), and that the unitary group of a factor of type $I_{\infty}$ or $I_{\infty}$ has essentially one closed invariant subgroup, viz. the (uniform) closure of the set of unitary operators which act as scalars on the complement of some subspace of finite relative dimension (all the other invariant subgroups are contained in this group and are constructed in a simple fashion-cf. Theorems 3 and 4). Among the various groups discussed one is shown to be topologically simple but not algebraically simple (i.e., it is shown to contain proper invariant subgroups but no such closed subgroups). In the final section a corollary on uniform approximation by certain classes of unitaries is derived from the foregoing results. This section concludes with a discussion of the problems arising in connection with the proved results and an indication of the possible extensions.

2. Auxiliary results. We begin this section with a theorem which is, in the main, superseded by the theorems in $\$ 3$. The later theorems give precise information concerning the invariant subgroups of the unitary group of a factor, whereas Theorem 1 deals only with the possibility of finding invariant subgroups with a compact factor group. Although the theorem below gives no new information in the case of finite factors, it adds slightly to our knowledge of $G_{f}$ (cf. Definition 1) in infinite factors. It is used principally in connection with the proof of Lemma 4, in which case, however, only the nonexistence of characters is needed. This fact, by itself, was proved in [2].

THEOREM 1. The unitary group $\mathcal{X}_{u}$ of a factor $\mathcal{X}$, not of type $\mathbf{I}_{n}$ (n finite), 
admits no nontrivial, uniformly continuous, finite-dimensional, unitary representation.

Proof. We shall show that a continuous, $n$-dimensional, unitary representation $\phi$ takes operators of the form $\lambda E+I-E,|\lambda|=1, E$ a projection in $\mathcal{X}$, into the identity matrix. This will complete the proof, for the group generated by these unitary operators lies dense in the group $\mathcal{X}_{u}$ (by the spectral representation theorem).

We observe first that if $E$ is held fixed and $\lambda$ varies $(|\lambda|=1)$, then $\phi(U(\lambda))$ is an abelian group of unitary matrices, where $U(\lambda)=\lambda E+I-E$, and, as such, is simultaneously reducible to diagonal form. The map from each reduced matrix to a particular diagonal coefficient provides a character on the $U(\lambda)$ and hence of $\lambda$. This character is continuous by assumption on $\phi$, and therefore has the form $\lambda \rightarrow \lambda^{m}$ ( $m$ some integer). Thus $\phi(U(\lambda))$ $=\operatorname{diag}\left\{\lambda^{m_{1}}, \cdots, \lambda^{m_{n}}\right\}$ (in reduced form) for all $\lambda$ such that $|\lambda|=1$. If $D(F)=D(E)$ and $D(I-F)=D(I-E)$, then $V(\lambda)=\lambda F+I-F$ is equivalent $\left(^{4}\right)$ to $U(\lambda)$, and thus, in reduced form (new basis), $\phi(V(\lambda)$ ) $=\operatorname{diag}\left\{\lambda^{m_{1}}, \cdots, \lambda^{m_{n}}\right\}$. The numbers $m_{i}$ depend only upon $D(E)=\alpha$ and $D(I-E)=\beta$ (i.e., the set of numbers $m_{i}$ depends only upon $\alpha, \beta$, for a given basis, not their arrangement). We write $m_{i}(\alpha, \beta)$ for $m_{i}$.

We consider two cases simultaneously, $\alpha \leqq 1 / n$ ! when $\mathcal{X}$ is of type $\mathrm{II}_{1}$ and $\alpha=\beta=\infty$ when $\mathcal{X}$ is an infinite factor. Suppose that in both these cases we have shown $m_{i}(\alpha, \beta)=0, i=1, \cdots, n$. Then, in the infinite case, if $P$ is infinite but $I-P$ is finite, we have $\lambda P+I-P=\left[\lambda\left(P-E_{1}\right)+I-P+E_{1}\right]$ - $\left[\lambda E_{1}+I-E_{1}\right]$ where $E_{1}$ and $P-E_{1}$ are infinite. Thus by assumption $\phi(\lambda P+I-P)=\phi\left[\lambda\left(P-E_{1}\right)+I-P+E_{1}\right] \phi\left[\lambda E_{1}+I-E_{1}\right]=I$. Hence, in this case, if $P_{1}$ is finite, $\phi\left(\lambda P_{1}+I-P_{1}\right)=\phi(\lambda I) \phi\left(\bar{\lambda}\left(I-P_{1}\right)+P_{1}\right)=I$, and in all events $\phi(\lambda E+I-E)=I$. In the $\mathrm{II}_{1}$ case, if $P$ is an arbitrary projection, choose $s$ orthogonal equivalent projections $P_{1}, \cdots, P_{8}$ with sum $P$ and each of dimension not exceeding $1 / n !$. We have $I=\phi\left[\left(\lambda P_{1}+I-P_{1}\right) \cdots\right.$ $\left.\left(\lambda P_{s}+I-P_{s}\right)\right]=\phi(\lambda P+I-P)$. It remains to prove that $m_{i}(\alpha, \beta)=0$ in the two cases mentioned.

Choose $k>\max _{i}\left\{\left|m_{i}(\alpha, \beta)\right|\right\}$, and let $E$ be a projection of dimension $n ! \alpha$ with $I-E$ infinite if $\alpha=\infty$ (we make the obvious conventions about $n ! \infty$, $\infty / k$, etc.). We also choose $n ! k$ equivalent, orthogonal projections $E_{1}, \cdots$, $E_{n ! k}$ with sum $E$. Since the $E_{i}$ 's all have the same dimension $\alpha / k$ and codimension $\gamma$, the $\phi\left(U_{i}(\lambda)\right)$ have the same $m_{i}$ (as sets), where $U_{i}(\lambda)=\lambda E_{i}+I$ $-E_{i}$. Now the $U_{i}(\lambda)$ all commute $(i=1, \cdots, n ! k ;|\lambda|=1)$ so that the $\phi\left(U_{i}(\lambda)\right)$ may be simultaneously brought to diagonal form. There are only $n$ ! possible arrangements of the $n$ numbers, $m_{j}(\alpha / k, \gamma), j=1, \cdots, n$, and each $\phi\left(U_{i}(\lambda)\right)$ corresponds to some such arrangement. Since there are $n ! k U_{i}(\lambda)$ 's,

(4) We say that two unitary operators in a factor are equivalent when it is possible to transform one into the other by means of a unitary operator in the factor. We use the phrase "equivalent projections" in the standard manner defined in R.O. I. 
at least $k$ of the $\phi\left(U_{i}(\lambda)\right)$ 's are the same. Renumber these as $\phi\left(U_{1}(\lambda)\right), \cdots$, $\phi\left(U_{k}(\lambda)\right)$, and let their diagonal form be $\operatorname{diag}\left\{\lambda^{m_{1}(\alpha / k, \gamma)}, \cdots, \lambda^{m_{n}(\alpha / k, \gamma)}\right\}$. But $U_{1}(\lambda) \cdots U_{k}(\lambda)=V(\lambda)=\lambda F+I-F$, where $F=E_{1}+\cdots+E_{k}$, so that $\phi\left(U_{1}(\lambda)\right) \cdots \phi\left(U_{m}(\lambda)\right)=\phi(V(\lambda))$ for all $\lambda$, and thus $k m_{j}(\alpha / k, \gamma)=m_{\tau(j)}(D(F)$, $D(I-F))=m_{\tau(j)}(\alpha, \beta)$, where $j \rightarrow \tau(j)$ is a permutation of the numbers $1, \cdots, n$. However, $k>\max _{j}\left\{\left|m_{j}(\alpha, \beta)\right|\right\} \geqq\left|m_{\tau(j)}(\alpha, \beta)\right|, j=1, \cdots, n$; and $k, m_{j}(\alpha / k, \gamma), m_{\tau(j)}(\alpha, \beta)$ are integers. It follows that $m_{i}(\alpha, \beta)=0$, $i=1, \cdots, n$.

If, in the foregoing proof, $\mathscr{X}$ had been of type $\mathbf{I}_{n}$, the second paragraph of the proof would still apply. If we assume, further, that $\phi$ is a character (i.e., the representation is 1-dimensional) and let $U(\lambda)=\lambda E+I-E$ be the family of unitary operators corresponding to a fixed 1-dimensional projection $E$, then $\phi(U(\lambda))=\lambda^{m}$ (and $m$ is the same no matter what 1-dimensional projection is chosen). It follows then that $\phi$ is the $m$ th power of the determinant and we have proved:

LEMMA 1. Each (uniformly) continuous character of the unitary group of a factor of type $\mathrm{I}_{n}$ ( $n$ finite) is a power of the determinant.

With the aid of this lemma and the fact that $\mathcal{H}_{u} /\{\lambda I\}$ is simple when $\mathscr{X}$ is of type $I_{n}$ we prove:

Lemma 2. Each closed, normal, noncentral subgroup $G$ of $\mathcal{X H}_{u}$, the unitary group of a factor $\mathcal{X}$ of type $\mathbf{I}_{n}$, is the inverse image under the determinant map of a closed subgroup of $\{\lambda:|\lambda|=1\}$. In particular, each such subgroup $G$ contains the unitaries having determinant 1.

Proof. If $G=\mathscr{X}_{u}$, the result is trivial; if not, then $G$ does not contain( $\left.{ }^{5}\right)$ $\{\lambda I\}$, but $G \cup\{\lambda I\}=\mathcal{X}_{u}$. Now $G \cap\{\lambda I\}$ is a closed proper subgroup of $\{\lambda I\}$, say the $k$ th roots of unity, so that $\mathscr{X}_{u} / G=\{\lambda I\} / G \cap\{\lambda I\}$ is isomorphic to $\{\lambda I\}$ under the map induced by the homorphism "raising to the $k$ th power" of $\{\lambda I\}$ onto $\{\lambda I\}$. This, of course, gives rise to a nontrivial continuous character of $\mathcal{X}_{u}$ with kernel $G$. The description of all such characters given in Lemma 1 completes the proof.

We shall need the following simple lemma on topological groups.

Lemma 3. If $\mathcal{T}$ is a topological group, $\mathcal{N}$ a closed normal subgroup of $\mathcal{T}$, and $\mathcal{C}$ a compact subgroup of $\mathcal{T}$, then $\mathcal{N} \cup \mathcal{C}$ is a closed subgroup of $\mathcal{T}$.

Proof. Since $\mathcal{N}$ is normal, $\mathcal{N} \cup \mathcal{C}=\{n c: n$ in $\mathcal{N}, c$ in $\mathcal{C}\}$. Suppose $b$ is a limit point of $\mathcal{N} \cup \mathcal{C}$; we wish to show that $b$ is in $\mathcal{N} \cup \mathcal{C}$, i.e., that $\left(^{6}\right) b=n c$ or $b \bigodot \cap \mathcal{N} \neq \varnothing$. In fact, if $b \bigodot \cap \mathcal{N}=\varnothing$, we can find a neighborhood $V_{\alpha}(a)$ about each point $a$ in $b \mathcal{C}$ such that $V_{\alpha}(a) \cap \mathcal{N}=\varnothing$. For this $\alpha$ and $a$, find $\rho$ such

(5) If $G_{1}$ and $G_{2}$ are subgroups of a group $G$, we denote by $G_{1} \cup G_{2}$ the subgroup of $G$ generated by $G_{1}$ and $G_{2}$.

(6) We denote the null set by $\varnothing$. 
that if $x, y$ are in $V_{\rho}(c)$, then $x$ is in $V_{\alpha}(y)$, and consider the neighborhood $V_{\rho}^{-1}(a)$ (i.e., the set of points $x$ such that $a$ is in $V_{\rho}(x)$ ) about $a$. A finite number of these neighborhoods $V_{p_{1}}^{-1}\left(a_{1}\right), \cdots, V_{p_{n}}^{-1}\left(a_{n}\right)$ cover $b \mathcal{C}$. Choose $V_{\rho} \subset \bigcap_{i} V_{\rho_{i}}$, and choose $n c$ in $V_{\rho}(b)$. Then $n$ is in $V_{\rho}\left(b c^{-1}\right)$. But $b c^{-1}$ is in $b C$ and hence in some $V_{\rho_{i}}^{-1}\left(a_{i}\right)$ so that $a_{i}$ is in $V_{\rho_{i}}\left(b c^{-1}\right)$. Now $n$ is in $V_{\rho}\left(b c^{-1}\right)$ and a fortiori in $V_{\rho_{i}}\left(b c^{-1}\right)$, so that, by choice of $\rho_{i}, n$ is in $V_{\alpha_{i}}\left(a_{i}\right)$, contradicting the fact that $V_{\alpha_{i}}\left(a_{i}\right) \cap \mathcal{N}=\varnothing$. Thus $b \bigodot \cap \mathcal{N} \neq \varnothing$, and the proof is complete.

Lemma 4. If a property $\mathrm{p}$ is such that $G \cup\{\lambda I:|\lambda|=1\}$ has property $\mathrm{p}$ if the subgroup $G$ of $\mathcal{X}_{u}$ ( $\mathcal{X}$ not of type $\mathrm{I}_{n}, n$ finite) has property $\mathrm{p}$, and if the only closed normal subgroup having property $\mathrm{p}$ and containing $\{\lambda I\}$ is $\mathcal{X}_{u}$, then the only closed normal subgroup of $\mathcal{X}_{u}$ with property $\mathrm{p}$ is $\mathcal{X}_{u}$.

Proof. If $G$ is a closed normal subgroup of $\mathcal{X}_{u}$ having property $\mathrm{p}$, then $G \cup\{\lambda I\}$ has property $\mathrm{p}$, by hypothesis, and is a closed normal subgroup of $\mathscr{X}_{u}$, by Lemma 3. Thus, by assumption, $G \cup\{\lambda I\}=\mathcal{X}_{u}$. But then $\mathcal{X}_{u} / G$ $=\{\lambda I\} / G \cap\{\lambda I\}$ and $\{\lambda I\} / G \cap\{\lambda I\}$, being locally compact and abelian, has nontrivial characters, unless $\{\lambda I\}=G \cap\{\lambda I\}$. Since $\mathcal{X}_{u}$, itself, has no nontrivial finite-dimensional representations, by Theorem 1 , it follows that $\{\lambda I\}=G \cap\{\lambda I\}$, so that $\mathscr{H}_{u}=G \cup\{\lambda I\}=G$.

In our applications of this lemma, $p$ will be the property of not being a subgroup of some fixed group.

The following lemma is an altered version of Lemma 3.2.3, R.O. II, suitable for our purposes.

Lemma 5. If $\mathcal{X}$ is a factor, $A$ is a normal operator in $\mathcal{X}$, and $F$ is a finite projection which commutes with $A$, then there exists a smaller projection in $\mathcal{X}$ which commutes with $A$ and which has any given dimension (not exceeding $D(F)$ ) in the range of the dimension function $D$ on $\mathcal{X}$.

Proof. The factor $\mathcal{X}_{F}$ (cf. R.O. I, pp. 186-188) is a finite factor. If it is discrete, it is all bounded operators on some finite-dimensional space, and the result is trivial. If it is continuous, the result follows by application of Lemma 3.2.3, R.O. II (this lemma applies to positive definite operators, but we can express our (restricted) normal operator $A F$ as a bounded Baire function of a positive definite operator in $\mathcal{X}_{F}$ ).

3. The closed normal subgroups of the unitary groups. We begin this section with the lemma which links the investigations of the unitary groups of the various types of factors. It is, so to speak, the common concluding portion of each of these investigations.

To say that a subgroup $G$ is a closed normal subgroup of $\mathscr{X}_{u}$ implies, in particular, that, along with $W, G$ contains $W V^{*} W V$ for all $V$ in $\mathcal{X}_{u}$. The wide choice of $V$ this totally noncommutative situation affords us makes $W V^{*} W V$ a quite arbitrary unitary, so arbitrary, indeed, that only the distinction between finite and infinite can prevent $G$ from being all of $\mathcal{X}_{u}$. On 
the other hand, it is a difficult matter to capitalize on this arbitrariness (at least in the case of the nondiscrete factors), and we are constrained, for the most part, to stay within reach of the spectral theorem by choosing $V$ in such a manner that $V^{*} W V$ commutes with $W$. This tactic alone would not suffice to give the final results concerning normal subgroups of $\mathfrak{x}_{u}$. In the next lemma, $W V^{*} W V$ is studied in a situation where it is possible to compute with finite $(2 \times 2)$ matrices.

LEMma 6. If $\mathcal{X}$ is a factor, not of type $\mathrm{I}_{n}$, $n$ finite, $\mathcal{X}_{u}$ the group of unitary operators in the factor, $W=\lambda E+I-E,|\lambda|=1, \lambda \neq 1$ a unitary operator in $\mathcal{X}$ with $E$ equivalent to $I-E$, and $G$ a closed normal subgroup of $\mathcal{X}_{u}$ containing $\lambda E+I-E$ and $\left\{\lambda^{\prime} I:\left|\lambda^{\prime}\right|=1\right\}$, then $G=\mathcal{X}_{u}$.

Proof. We show first that our group $G$ contains all unitary operators of the form $\gamma E+I-E$, with $\gamma$ an arbitrary complex number of modulus 1 . In fact, let $V$ be a partial isometry from $\left(^{7}\right) E$ to $I-E$, and let $V(\theta)$ be the unitary operator

$$
(\sin \theta) E+(\cos \theta)\left(V+V^{*}\right)-(\sin \theta)(I-E) .
$$

If we let $W(\theta)=W V(\theta) W V(\theta)^{*}$, a simple computation shows that $T(W(\theta)$ ) assumes all values on the segment between $\lambda$ and $\left(\lambda^{2}+1\right) / 2$ as $\theta$ goes from 0 to $\pi / 2$ (although $\mathcal{X}$ may not have a trace, the operators $W(\theta)$ all lie in the type $\mathrm{I}_{2}$ factor generated by the $2 \times 2$ matrix units $E, I-E, V$, and $V^{*}$-it is with respect to this factor that we take the normalized trace, $T(W(\theta)))$. Now $W(\theta)$ has spectrum consisting of two numbers $\alpha, \beta$ (not necessarily distinct); the eigenspace of each is equivalent to $E$ (if they are distinct). Moreover, $\alpha \beta=\lambda^{2}, T(W(\theta))=(\alpha+\beta) / 2$ (so that $\alpha=\beta$ for only a finite set of values), and the map from the set of unordered pairs $(\alpha, \beta)$ to $T(W(\theta))$ is one-one (i.e., the spectrum of a $2 \times 2$ matrix is determined by its trace and determinant). Since $T(W(\theta))$ varies over an uncountable set $(\lambda \neq 1)$, at least one of $\alpha, \beta$ is not a root of unity, say $\alpha$, and they are distinct, for some $\theta_{1}$. We write $W\left(\theta_{1}\right)=\alpha F+\beta(I-F)$. Then $\left[W\left(\theta_{1}\right)\right]^{n}=\alpha^{n} F+\beta^{n}(I-F)$ is in $G$ and $\left\{\alpha^{n}\right\}$ lies dense in the complexes of modulus 1 . We shall be through with the proof that $G$ contains all $\gamma F+I-F,|\gamma|=1$, if we can show that $G$ contains some $\eta F+I-F$ with $\eta$ not a root of unity; for then $G$ contains $\left(\eta^{m}\right) F+I-F$, $m$ an integer, and these operators lie dense in all $\gamma F+I-F$, since $\left\{\eta^{m}\right\}$ lies dense on the unit circle. The same argument would apply to $\gamma E+I-E$, $|\gamma|=1$, if $\lambda$ were not a root of unity, so that we may assume that $\lambda$ is an $n$th root of unity. Given $\tau$ on the unit circle, we can clearly find an integer $k$ between 0 and $n$ such that $\tau F+\left(\lambda^{k} / \tau\right)(I-F)$ is a limit point of $\left[W\left(\theta_{1}\right)\right]^{m}$ $=\alpha^{m} F+\left(\lambda^{2 m} / \alpha^{m}\right)(I-F)$. Thus $\tau F+\left(\lambda^{k} / \tau\right)(I-F)$, and hence $\left(\tau / \lambda^{k}\right)(\tau F$ $\left.+\left(\lambda^{k} / \tau\right)(I-F)\right)=\left(\tau^{2} / \lambda^{k}\right) F+(I-F)$ is in $G$. It is clearly possible to choose $\tau$

(7) We use the same symbol to denote both the projection and its range. The context will make clear which is meant in any given instance. 
on the unit circle so that $\tau^{2} / \lambda^{k}$ is not a root of unity no matter what integer, between 0 and $n, k$ is. Thus $G$ contains all unitary operators of the form $\gamma F+I-F,|\gamma|=1$, and, since $G$ is normal, it contains all (equivalent) operators of the form $\gamma E+I-E$ with $E$ equivalent to $I-E$. If $\mathcal{X}$ is an infinite factor and $P$ is an infinite projection in $\mathcal{X}$ with $I-P$ finite, we write $\lambda P+I$ $-P=\left[\lambda\left(P-E_{1}\right)+I-P+E_{1}\right]\left[\lambda E_{1}+I-E_{1}\right]$, where $E_{1}$ is an infinite projection in $P$ with $P-E_{1}$ infinite. Thus $\lambda P+I-P$ is in $G$. If $P$ is finite and $I-P$ is infinite, then $\lambda P+I-P=(\lambda I)[\bar{\lambda}(I-P)+P]$ is in $G$. If $\mathcal{X}$ is a factor of type $\mathrm{II}_{1}$ and $P$ is a projection in $\mathcal{H}$ such that $P$ is equivalent to a subprojection of $I-P$, we choose $\zeta$ an $n$th root of unity and $n$ orthogonal equivalent projections $F_{1}, \cdots, F_{n}$ of dimension $D(P) / n$ in $F$. Let $F^{\prime}$ be a projection in $I-F$ equivalent to $F-F_{i}$, under a partial isometry $U_{i}^{\prime}$. Let $U_{i}$ be the unitary operator which is $U_{i}^{\prime}$ on $F-F_{i}, U_{i}^{\prime *}$ on $F^{\prime}$, and the identity on $I-\left(F+F^{\prime}-F_{i}\right)$. The unitary operator

$$
U=\prod_{i=1}^{n} U_{i}^{*}(\zeta F+I-F) U_{i}=\zeta\left(\sum_{i=1}^{n} F_{i}\right)+I-\sum_{i=1}^{n} F_{i}
$$

is in $G$ and is equivalent to $\zeta P+I-P$. Thus $\zeta P+I-P$ is in $G$ for all roots of unity $\zeta$, and all projections $P$ equivalent to a subprojection of their complement $I-P$. If, on the other hand, $I-P$ is equivalent to a subprojection of $P$, then $\zeta(\zeta(I-P)+P)=\zeta P+I-P$ is in $G$. Since the roots of unity are dense in the complexes of modulus 1 , and $G$ is closed, $\gamma P+I-P$ is in $G$ for all $\gamma,|\gamma|=1$, and all projections $P$ in $\mathscr{X}$. Thus, by the spectral theorem and the fact that $G$ is closed, we have $G=\mathcal{X}_{u}$.

We prove now the first main theorem.

THEOREM 2. If $\mathcal{X}$ is a factor of type $\mathrm{II}_{1}$ or III and $\mathcal{X}_{u}$ is the group of unitary operators in $\mathcal{X}$, then the only proper, closed, invariant subgroups of $\mathcal{H}_{u}$ are the subgroups of the center $\{\lambda I:|\lambda|=1\}$.

Proof. By application of Lemma 4, with property $\mathrm{p}$ the property of not being a subgroup of $\{\lambda I\}$, we see that it suffices to consider groups containing $\{\lambda I\}$. Suppose, then, that $G$ is a closed, invariant subgroup of $\mathcal{X}_{u}$ containing $\{\lambda I\}$ properly, and let $U$ be an operator in $G$ not in $\{\lambda I\}$. Since the scalars are in $G$, we can assume that 1 is in the spectrum of $U$. If -1 is the only other point in the spectrum of $U$, then $U=-E+(I-E)$; and a modified version of the last paragraph of the proof of Lemma 6 shows that we may assume even that $E$ is equivalent to $I-E$. But then, by Lemma 6 , $G=\mathscr{X}_{u}$. We assume that $U$ has a point $\alpha \neq 1,-1$ in its spectrum. Let $\epsilon>0$ and $\alpha^{2} P+I-P$, with $P$ equivalent to $I-P$, be given. Let $Q_{1}, Q_{2}$ be spectral projections for $U$ corresponding to the arcs cut off by the closed disks of radius $\delta$ about 1 and $\alpha$, respectively. Choose a positive integer $m$ so that $1 / 2 m \leqq \min \left[D\left(Q_{1}\right), D\left(Q_{2}\right)\right]$ (if $\mathcal{X}$ is of type III, we choose $m=0$ and agree 
that $1 / 0=\infty)$, and let $E_{1}$ and $F_{1}$ be projections in $Q_{1}, Q_{2}$ respectively, each of dimension $1 / 2 m$ and commuting with $U$ (see Lemma 5). Let $V_{1}$ be a partial isometry from $E_{1}$ to $F_{1}$, and let $V$ be the unitary operator which is $V_{1}$ on $E_{1}$, $V_{1}^{*}$ on $F_{1}$, and the identity on $I-\left(E_{1}+F_{1}\right)$. Then $W=U^{*} V^{*} U V$ is in $G$ and is close to $\alpha E_{1}+\bar{\alpha} F_{1}+I-\left(E_{1}+F_{1}\right)$, for both $W$ and $\alpha E_{1}+\bar{\alpha} F_{1}+I-\left(E_{1}+F_{1}\right)$ are the identity on $I-\left(E_{1}+F_{1}\right), U^{*}$ is close to 1 and $V^{*} U V$ is close to $\alpha$ on $E_{1}$, and $U^{*}$ is close to $\bar{\alpha}$ and $V^{*} U V$ is close to 1 on $F_{1}$ (for small $\delta$ ). Let $E_{2}, \cdots, E_{m} ; F_{2}, \cdots, F_{m}$ be $2 m-2$ orthogonal equivalent projections with sum $I-\left(E_{1}+F_{1}\right)$ (each of dimension $\left.1 / 2 m\right)$ and let $U_{i}, V_{i}(i=2, \cdots, m)$ be partial isometries from $E_{1}$ to $E_{i}$ and $F_{1}$ to $F_{i}$ respectively. If $X_{i}$ is the unitary operator which is $U_{i}$ on $E_{1}, U_{i}^{*}$ on $E_{i}, V_{i}$ on $F_{1}, V_{i}^{*}$ on $F_{i}$, and the identity on $I-\left(E_{1}+E_{i}+F_{1}+F_{i}\right)$, then $Y=\prod_{i=1}^{m} X_{i}^{*} W X_{i}$ is in $G$ and (for small $\delta$ )

$$
\left\|Y-\left(\alpha \sum_{i=1}^{m} E_{i}+\bar{\alpha} \sum_{i=1}^{m} F_{i}\right)\right\|<\epsilon .
$$

Now $E=\sum_{i=1}^{m} E_{i}$ is equivalent to $F=\sum_{i=1}^{m} F_{i}$, so that $\alpha E+\bar{\alpha} F$ is equivalent to $\alpha P+\bar{\alpha}(I-P)$. It follows that $\alpha P+\bar{\alpha}(I-P)$ is approximable to within $\epsilon$ by an operator in $G$, so that $\alpha P+\bar{\alpha}(I-P)$ and $\alpha(\alpha P+\bar{\alpha}(I-P))=\alpha^{2} P$ $+(I-P)$ are in $G$. An application of Lemma 6 completes the proof.

Definition 1. A number $\alpha$ in the spectrum of a normal operator $C$ in a factor $\mathcal{X}$ is said to be "a center of infinite density" if the spectral projection of $C$ corresponding to any open set about $\alpha$ has infinite relative dimension.

Strictly speaking, we should use the phrase "a center of infinite density relative to $\mathscr{X}$ " to describe $\alpha$. Indeed, if $A$ is a normal operator in a factor $\mathscr{X}$ of type $\mathrm{II}_{1}$, then no point of the spectrum is a center of infinite density; whereas relative to the factor consisting of all bounded operators, every point of the spectrum is a center of infinite density. However, we shall maintain the terminology of Definition 1, trusting to the context to make our meaning clear.

THEOREM 3. If $\mathcal{X}$ is a factor of type $\mathrm{I}_{\infty}$ or $\mathrm{II}_{\infty}$, the set $G_{\text {s }}$ of all unitary operators in $\mathcal{X}$ with exactly one center of infinite density is a closed, proper, normal subgroup of $\mathcal{X}_{u}$, the group of unitary operators in $\mathcal{X}$.

We shall need the following lemma for the proof of this theorem.

Lemma 7. If $\mathcal{X}$ is an arbitrary factor and $P$ and $Q$ are projections in $\mathscr{H}$ such that $D(Q)>D(I-P)$, then $P \cap Q$, the intersection of $P$ and $Q$, is not 0 .

Proof. The assumption $D(Q)>D(I-P)>0$ implies, in particular, that $D(I-P) \neq \infty$. Thus we can choose a finite projection $Q_{1}$ in $Q$ such that $D\left(Q_{1}\right)>D(I-P)$. Let $E=Q_{1} \cup(I-P)$. By R.O. I, Lemma 7.3.5, $E$ is a finite projection. We have: 


$$
\begin{aligned}
D(E) & +D\left[Q_{1} \cap(E-(I-P))\right] \\
& \geqq D\left[Q_{1} \cup(E-(I-P))\right]+D\left[Q_{1} \cap(E-(I-P))\right] \\
& =D\left(Q_{1}\right)+D[E-(I-P)]>D(I-P)+D[E-(I-P)]=D(E) .
\end{aligned}
$$

Thus $D\left[Q_{1} \cap(E-(I-P))\right]>0$. Since $Q_{1}$ is contained in $Q,(E-(I-P))$ is contained in $P$, and $Q_{1} \cap(E-(I-P))$ is nonzero; $Q \cap P \neq 0$.

Proof of Theorem 3. We prove this result by showing that $G_{f}^{\prime}$, the set of all unitary operators in $\mathcal{H}$ which act as $\lambda I$ on the complement of some subspace of finite relative dimension, is an invariant subgroup of $\mathcal{X}_{u}$, and that $G_{f}$ is its closure (8). If $U_{1}, U_{2}$ are in $G_{f}^{\prime}$ and $E_{1}, E_{2}$ are finite projections on the complements of which $U_{1}, U_{2}$ are $\lambda_{1} I, \lambda_{2} I$, respectively, then $U_{1}^{-1} U_{2}$ is $\bar{\lambda}_{1} \lambda_{2} I$ on the complement of $E_{1} \cup E_{2}$, a finite projection. Thus $U_{1}^{*} U_{2}$ is also in $G_{f}^{\prime}$, which is therefore a subgroup of $\mathcal{H}_{u}$. Clearly $G_{f}^{\prime}$ is an invariant subgroup of $\mathcal{X}_{u}$.

Every normal operator $C$ in $\mathscr{X}$ has at least one center of infinite density in each closed subset of the spectrum whose corresponding spectral projection has infinite relative dimension; for otherwise we could cover each point of the set with an open set whose spectral projection is finite, and, by compactness, we could cover the entire set with a finite number of these sets. The spectral projection for this closed set would then be contained in a finite union of finite projections. But the former projection is assumed to be infinite and the latter projection is finite. It follows, in particular, from this that each normal operator in $\mathcal{X}$ has at least one center of infinite density, for the spectral projection corresponding to the total spectrum is $I$.

If, now, $U$ is in $G_{f}$ and $\alpha$ is the unique center of infinite density for $U$, then $\| U-\left(\alpha F_{\epsilon}+\left(I-F_{\epsilon}\right) U \| \leqq \epsilon\right.$, where $F_{\epsilon}$ is the spectral projection for the open arc $\Gamma$ of the unit circle cut off by the circle of radius $\epsilon$ about $\alpha$ (by the spectral representation theorem). The projection $I-F_{\epsilon}$ is the spectral projection for the complement $\Gamma^{\prime}$ of the arc $\Gamma$ on the unit circle. If $D\left(I-F_{\epsilon}\right)$ were infinite, $\Gamma^{\prime}$ would contain a center of infinite density for $U$, by the result of the preceding paragraph. This would contradict the uniqueness of $\alpha$, so that $D\left(I-F_{\epsilon}\right)$ is finite and $\alpha F_{\epsilon}+\left(I-F_{\epsilon}\right) U$ is in $G_{f}^{\prime}$. It follows that $U$ is in the closure of $G_{f}^{\prime}$ and thus $G_{f}$ is contained in the closure of $G_{f}^{\prime}$.

Suppose $V$ in $\mathcal{X}_{u}$ has at least two distinct centers of infinite density $\beta, \gamma$. We assert that $\|V-W\| \geqq|\beta-\gamma| / 2$ for all $W$ in $G_{f}^{\prime}$. Indeed, let $\epsilon>0$ be assigned, and let $E$ be a subspace on which $W$ acts as $\lambda I$ and whose complement has finite relative dimension. If $F_{\epsilon}$ is the spectral projection defined for $V$ as it was for $U$ above with $\beta$ replacing $\alpha$, then, since $\beta$ is a center of infinite density, $D\left(F_{\epsilon}\right)=\infty>D(I-E)$. Hence, by Lemma $7, E \cap F_{\epsilon} \neq 0$. Let

(8) We are indebted to I. Singer for a stimulating conversation at the most preliminary stage of this investigation. It was Singer who pointed out the existence of the normal subgroup $G_{f}^{\prime(1)}$ in the $I_{\infty}$ case (see the definition following the proof of Theorem 3 ). 
$x$ be a unit vector in the range of $E \cap F_{\epsilon}$; then

$$
\|V x-\beta x\|=\left\|V F_{\epsilon} x-\beta F_{\epsilon} x\right\| \leqq\left\|V F_{\epsilon}-\beta F_{\epsilon}\right\| \leqq \epsilon,
$$

moreover $W x=\lambda x$. Thus

$$
\begin{aligned}
\|W-V\| & \geqq\|W x-V x\|=\|W x-\beta x+\beta x-V x\| \\
& \geqq\|\lambda x-\beta x\|-\|\beta x-V x\| \geqq|\lambda-\beta|-\epsilon
\end{aligned}
$$

for all $\epsilon>0$, so that $\|W-V\| \geqq|\lambda-\beta|$. By symmetry, $\|W-V\| \geqq|\lambda-\gamma|$, and thus $2\|W-V\| \geqq|\gamma-\lambda|+|\lambda-\beta| \geqq|\gamma-\beta|$. We conclude from this that $V$ is not in the closure of $G_{f}^{\prime}$ and hence $G_{f}$ is exactly the closure of $G_{f}^{\prime}$.

The following theorem completes the determination of the closed normal subgroups of the infinite factors. Before stating the theorem, we introduce the following notation: $G_{f}{ }^{(1)}$ is the subgroup of $G_{f}$ consisting of those unitary operators in $\mathcal{X}$ whose unique center of infinite density is $1 ; G_{f}^{\prime(1)}$ is the subgroup of $G_{f}^{\prime}$ consisting of those unitary operators in $\mathcal{X}$ which act as the identity on the complement of a subspace of finite relative dimension.

THEOREM 4. If $\mathcal{X}$ is a factor of type $\mathrm{I}_{\infty}$ or $\mathrm{II}_{\infty}$ and $\mathcal{X}_{u}$ is its unitary group, then the only proper closed normal subgroups of $\mathcal{X}_{u}$ are the closed normal subgroups of $G_{f}$. The closed normal subgroups of $G_{f}$ are those generated by $G_{f}^{(1)}$ and the finite subgroups of $\{\lambda I:|\lambda|=1\}$, and these finite subgroups themselves.

Proof. In this case, we take property $\mathrm{p}$ to be the property of not being a subgroup of $G_{f}$, and again we see that it suffices to consider closed normal subgroups containing $\{\lambda I\}$. Suppose that $G$ is such a subgroup and that $G$ is not contained in $G_{f}$. Choose $U$ in $G$ but not in $G_{f}$ and let $1, \alpha$ be two of its centers of infinite density (since $U$ is not in $\mathcal{G}_{f}$, we know that it has at least two centers of infinite density, and, since the scalars are in $G$, we may assume that one of these centers is 1). If possible, we choose $\alpha$ distinct from -1 . Assume first that $\alpha \neq-1$, and let $P$ be a projection in $\mathcal{X}$ such that $P$ is equivalent $I-P$. Let $\epsilon>0$ be assigned. We shall approximate the unitary $\alpha^{2} P+I-P$ to within $\epsilon$ uniformly by operators in $G$. Since $G$ is closed, it will follow from this that $\alpha^{2} P+I-P$ is in $G$, and hence, by Lemma 6 , that $G=\mathcal{X}_{u}$ (then, of course, we must deal with the case where $\alpha=-1$ ).

Let $E$ and $F$ be the spectral projections for $U$ of the open $\operatorname{arcs} \Gamma_{1}, \Gamma_{2}$ cut off from the unit circle by small circles of radius $\delta$ about 1 and $\alpha$ respectively. Since 1 and $\alpha$ are centers of infinite density for $U, D(E)=D(F)=\infty$. Let $V_{1}$ be a partial isometry from $E$ to $F$ and let $V$ be the unitary operator which is $V_{1}$ on $E, V_{1}^{*}$ on $F$, and the identity on $I-(E+F)$. We write $W=U^{*} V^{*} U V$. If possible choose $\delta$ so small that $I-(E+F)$ is infinite. In this case, let $P_{1}, P_{2}$ be orthogonal equivalent projections of infinite relative dimension such that $P_{1}+P_{2}=I-(E+F)$, and let $U_{1}, U_{2}$ be partial isometries from $E$ to $P_{1}$ and 
$F$ to $P_{2}$, respectively. If $X$ is the unitary operator which is $U_{1}$ on $E, U_{1}^{*}$ on $P_{1}, U_{2}$ on $F$, and $U_{2}^{*}$ on $P_{2}$, then

$$
\left\|\alpha W X^{*} W X-\alpha^{2}\left(E+P_{1}\right)-\left(P_{2}+F\right)\right\|<\epsilon
$$

( $\delta$ chosen suitably small, dependent on $\epsilon$-this inequality follows from the spectral representation theorem). Now $\alpha W X^{*} W X$ is in $G$ and $\alpha^{2}\left(E+P_{1}\right)$ $+\left(P_{2}+F\right)$ is equivalent to $\alpha^{2} P+I-P$. Thus the same inequality holds for a suitable conjugate of $\alpha W X^{*} W X$ and $\alpha^{2} P+I-P$. We suppose now that it is not possible to choose $\delta$ so that $I-(E+F)$ is infinite (or equivalently, that $\alpha$, 1 are the only centers of infinite density for $U$ ). The ensuing argument also handles the case $\alpha=-1$ (this case was forced on us by the fact that $-1,1$ were the only two centers of infinite density). In this situation, the spectral projection for $U$ corresponding to any closed arc of the unit circle which contains neither 1 nor $\alpha$ has finite relative dimension. If 1 and $\alpha$ are the only points in the spectrum of $U$, then, by Lemma $6, G=\mathcal{X}_{u}$, and we are through. If not, let $\lambda$ be another point of the spectrum of $U$, and let $F_{1}$ be a spectral projection for $U$ corresponding to a small closed arc about $\lambda$. We have $D\left(F_{1}\right) \neq \infty$. Let $\tau$ be a point very near 1 , and consider small arcs starting at $\tau$ and going towards 1 (we choose $\tau$ so that the small closed arc $(\tau, 1)$ has infinite relative dimension). The spectral projections for $U$ corresponding to these arcs have a finite relative dimension which tends toward $\infty$, unless 1 is in the point spectrum of $U$ and has an eigenspace of infinite relative dimension. In any event, we can choose a projection $E_{1}$ which commutes with $U$ and has relative dimension $D\left(F_{1}\right)$, in a spectral projection with relative dimension greater than $D\left(F_{1}\right)$ corresponding to one of the arcs, by Lemma 5 , in one case, and in the eigenspace of 1 , in the other. Let $W_{1}$ be a partial isometry from $F_{1}$ to $E_{1}$, and let $W^{\prime}$ be the unitary which is $W_{1}$ on $F_{1}, W_{1}^{*}$ on $E_{1}$, and the identity on $I-\left(E_{1}+F_{1}\right)$. Then $U^{*} W^{\prime *} U W^{\prime}$ is the identity on $I-\left(E_{1}+F_{1}\right)$, close to $\lambda$ on $E_{1}$, and close to $\bar{\lambda}$ on $F_{1}$. Let $P_{1}$ be a finite projection contained in $I-\left(E_{1}+F_{1}\right)$. It follows that $U^{*} W^{*} U W^{\prime}$ is the identity on $I-\left(E_{1}+F_{1}+P_{1}\right)$ and a unitary operator on $E_{1}+F_{1}+P_{1}$ which is not a multiple of $I$ (really, of $\left.E_{1}+F_{1}+P_{1}\right)$. Now the set $\mathcal{H}$ of unitary operators in $\mathcal{X}$ which act as the identity on $I-\left(E_{1}+F_{1}+P_{1}\right)$ is a group topologically isomorphic to the unitary group of the factor $\mathcal{X}_{\left(E_{1}+F_{1}+P_{1}\right)}$ (cf. R.O. I, pp. 186-188). Since $E_{1}+F_{1}+P_{1}$ is finite, $\mathscr{X}_{\left(E_{1}+F_{1}+P_{1}\right)}$, the restricted factor, is finite. If $\mathscr{X}$ is of type $I_{\infty}$, the restricted factor is of type $\mathrm{I}_{n}$; if $\mathcal{X}$ is of type $\mathrm{II}_{\infty}$, the restricted factor is a $\mathrm{II}_{1}$. Now the intersection of $G$ and $\mathcal{H C}$ is a closed invariant subgroup of $\mathcal{H}$ not contained in the center, by virtue of the foregoing construction. In the $\mathrm{II}_{1}$ case, it follows that $G$ contains $\mathfrak{H}$, by Theorem 2 ; and, in the $\mathrm{I}_{n}$ case, that $G$ contains the unitary operators in $\mathfrak{F}$ with determinant 1 (call this set $\mathfrak{F}_{\boldsymbol{s}}$ ), by Lemma 2 . By invariance, we see that $G$ contains all unitary operators which are the identity on the complement of a space of dimension $D\left(E_{1}+F_{1}+P_{1}\right)$ (and have determinant 1 on the finite space in the $\mathrm{I}_{n}$ case). 
Since $P_{1}$ was of arbitrary positive dimension, we may drop the restriction on the dimension of the finite subspace. We assert that the uniform closure of the set of unitaries in a $I_{\infty}$ having determinant 1 on a finite subspace and acting as the identity on the complement contains the set of unitaries which act as the identity on the complement of some finite-dimensional subspace. In fact, it suffices to show that this closure contains those unitaries which are $\lambda$ on a finite-dimensional subspace and the identity on the complement; for then this closure contains the sum of the scalars and the special unitary group (which is the full unitary group) on each finite subspace. Let $E_{0}$ be an $m$-dimensional subspace and let $\lambda,|\lambda|=1$, be given along with a small positive $\epsilon$. Suppose $\lambda^{m}=e^{i \theta}$. Choose an integer $k$ so large that $|\beta-1|<\epsilon$, where $\beta=e^{-i(\theta / k)}$. Let $F_{0}$ be a $k$-dimensional subspace of $I-E_{0}$, and consider the unitary operator $\lambda E_{0}+\beta F_{0}+I-\left(E_{0}+F_{0}\right)$. This unitary has determinant equal to $\lambda^{m} \beta^{k}=1$, moreover

$\left\|\lambda E_{0}+\beta F_{0}+I-\left(E_{0}+F_{0}\right)-\left(\lambda E_{0}+I-E_{0}\right)\right\|=\left\|\beta F_{0}-F_{0}\right\|=|\beta-1|<\epsilon$.

Thus $\lambda E_{0}+I-E_{0}$ is in the closure, and we have our assertion. It follows that $G$ contains the set of unitary operators which act as the identity on the complement of some finite-dimensional subspace whether $\mathcal{X}$ is of type $\mathrm{I}_{\infty}$ or $\mathrm{II}_{\infty}$.

With this information we return to the study of $U$. Let $\epsilon>0$ and $\alpha P+I-P$ be given, $P$ equivalent to $I-P$. Let $E$ and $F$ be as before, with $\delta<\epsilon$. By what we have just proved, $G$ contains $(I-E-F) U^{*}+E+F$ (since 1 and $\alpha$ are the only centers of infinite density for $U, I-E-F$ is finite). Thus $G$ contains $U\left[(I-E-F) U^{*}+(E+F)\right]=I-E-F+(E+F) U$. This last operator, however, is within $\epsilon$ of $\alpha F+I-F$ which is equivalent to $\alpha P+I-P$. Thus $\alpha P$ $+I-P$ is in $G$, and, by Lemma $6, G=\mathcal{X}_{u}$.

The argument which serves to show that $G$ contains $G_{f}^{(1)}$ shows that $G_{f}^{(1)}$ is topologically simple. In fact, if $U$ in $G_{j}^{(1)}$ is different from the identity, we can find $W$ in $G_{f}^{(1)}$ (in fact in $G_{f}^{\prime(1)}$ ) such that $W U W^{*} U^{*}$ is not a scalar on some finite-dimensional subspace and is the identity on the complement of this subspace, unless the spectrum of $U$ consists of 1 and -1 alone, in which case we can assert the same property for $U$ itself as we did for $W U W^{*} U^{*}$. Exactly as in the second preceding paragraph, we conclude that the closed normal subgroup of $G_{f}^{(\mathbf{1})}$ generated by $W U W^{*} U^{*}$ (or $U$, as the case may be) is all of $G_{f}^{(1)}$. Since $G_{f}$ is the direct sum of $G_{f}^{(1)}$ and $\{\lambda I:|\lambda|=1\}$, we have the fact that the $G_{f}^{(1)}$ coordinates of the elements of a closed normal subgroup $S$ of $G_{f}$ is a closed (since $\{\lambda I\}$ is compact) normal subgroup of $G_{f}^{(\mathbf{1})}$ and therefore either the identity or $G_{f}^{(1)}$. If it is the identity, then $S$ is a closed subgroup of $\{\lambda I\}$. Suppose it is $G_{f}^{(1)}$, and let $\phi$ be the map from $\{\lambda I\}$ to $G_{f}^{(1)}$ defined by $\lambda I \rightarrow U$ where $(U, \lambda I)$ is in $\delta$. The map $\phi$ is defined on that subgroup of $\{\lambda I\}$ which appears as coordinates in $S$ and is a homomorphism if it is single-valued (i.e., if $\left(U_{0}, \lambda I\right),\left(V_{0}, \lambda I\right)$ in $S$ implies $\left.U_{0}=V_{0}\right)$. But this 
would imply that $G_{f}^{(1)}$ is abelian, which is absurd. Suppose that $\left(U_{0}, \lambda I\right)$, $\left(V_{0}, \lambda I\right)$ both appear in $\delta$ with $U_{0} \neq V_{0}$; then $\left(U_{0} V_{0}^{*}, I\right)$ is in $\delta$ with $U_{0} V_{0}^{*}$ $\neq I$, so that the set of $W_{0}$ 's in $G_{f}^{(1)}$ such that $\left(W_{0}, I\right)$ is in $\delta$ contains more than the identity. This set is, however, a closed normal subgroup of $G_{f}^{(1)}$ and hence all of $G_{f}^{(1)}$. If $(U, \lambda I)$ is in $\mathcal{S}$, then $\left(U^{*}, I\right)(U, \lambda I)=\lambda I$ is in $\delta$, and thus $\delta$ is generated by $G_{f}^{(1)}$ and a closed subgroup of $\{\lambda I\}$ (closed, since $G_{f}^{(1)}$ and a nonclosed subgroup of $\{\lambda I\}$ generate a nonclosed subgroup of $G_{f}$ ).

It is interesting to note that $G_{f}^{(1)}$ is a (complete) topological group which while topologically simple is not algebraically simple $\left(G_{f}^{\prime(1)}\right.$ is a dense normal subgroup as well as the determinant 1 subgroup of $\left.G_{f}^{\prime(1)}\right)$.

4. Concluding remarks. It is a simple consequence of the theorems of the preceding section that each unitary operator in a factor is uniformly approximable by finite products of unitary operators chosen from almost any invariantly defined class of operators in the factor. More precisely we can state:

COROLlaRY 1. If $\mathcal{X}$ is a factor and $\mathrm{p}$ is a property of unitary operators which is invariant under unitary equivalence (by unitary operators in the factor) such that a unitary operator has property $\mathrm{p}$ only if its inverse has property $\mathrm{p}$ and such that some noncentral unitary operator of the factor has property $\mathrm{p}$ (in the case of factors of type $\mathrm{I}_{\infty}, \mathrm{II}_{\infty}$ we assume that some unitary operator not iu $G_{f}$ has property $\left.\mathrm{p}\right)$, then, for each unitary operator $U$ in $\mathcal{X}$ and each positive $\epsilon$, there exists a finite set of unitary operators $U_{1}, \cdots, U_{n}$ in $\mathcal{X}$ each with property $\mathrm{p}$ such that $\left\|U-U_{1} \cdots U_{n}\right\|<\epsilon$.

Proof. Under the given assumptions on $\mathrm{p}$, the set of all finite products of unitary operators in $\mathcal{H}$ having the property $\mathrm{p}$ is a normal noncentral subgroup of $\mathcal{X}_{u}$ (not contained in $G_{f}$, in the $\mathrm{I}_{\infty}, \mathrm{II}_{\infty}$ cases), and thus, by the theorems of $\S 3$, the uniform closure of this group must be all of $\mathcal{X}_{u}$.

One can conclude from this corollary, for example, that products of selfadjoint unitaries, or products of unitary operators with continuous spectrum, or products of unitary operators with three points in their spectrum, etc., lie uniformly dense in the group of all unitary operators in the factor.

It is appropriate to remark that, although we have made heavy use of the techniques and constructions of R.O. I, and R.O. I deals with separable Hilbert space, there is very little imagination needed to see what happens in the nonseparable case. The situation in the $\mathrm{II}_{1}$ case remains the same, and the proof given in this paper remains valid. More closed invariant subgroups appear in the case of infinite factors because of the now distinct equivalence classes of infinite projections.

The situation becomes quite complicated when we pass to arbitrary rings of operators. Keeping in mind von Neumann's reduction theory [7], we may for the moment conceive of the arbitrary ring as a direct sum of factors. Even if we deal only with the case of rings purely of type $\mathrm{II}_{1}$ (cf. [4]), we 
have to contend with all the complications inherent in taking an algebraic direct sum of simple groups. To add to these difficulties, there are the measurability problems which must be dealt with if the general results of [7] are to be applied. It is likely, in view of these considerations, that there is nothing very elegant which can be said in the general case. In connection with [4], it should be mentioned that the results we obtain are applicable to Kaplansky's $A-W^{*}$ algebras (the central ones).

As far as the topological results are concerned, those we present are the strongest possible (it is a simple matter to deal with the weaker topologies, for the groups then are, in particular, uniformly closed). A problem which seems to be of a higher order of difficulty (and interest) than the extensions indicated above is the question of the algebraic nature of the unitary groups considered. Specifically, the question of whether or not the unitary group of a factor of type $\mathrm{II}_{1}$ is algebraically simple seems particularly important because of the Lie group, Lie algebra connections mentioned in the introduction. One can probably clear up the algebraic situation in the case of factors of type $I_{\infty}$, and, in fact, Kakutani has recently communicated to us the fact that each unitary operator in a factor of type $I_{\infty}$ can be expressed as a product of four self-adjoint unitary operators in the factor (Halmos has shown that $\omega I$ can't be expressed as a product of three such unitary operators, where $\omega$ is a primitive cube root of unity). The essential features of Kakutani's proof are such, however, that no light is thrown on the $\mathrm{II}_{1}$ case.

\section{BiBLIOGRAPHY}

1. B. Fuglede and R. V. Kadison, On determinants and a property of the trace in finite factors, Proc. Nat. Acad. Sci. U.S.A. vol. 37 (1951) pp. 425-431.

2. - Determinant theory in finite factors, to appear in Ann. of Math.

3. I. Gelfand and M. Neumark, On the imbedding of normed rings into the ring of operators in Hilbert space, Rec. Math. (Mat. Sbornik) N.S. vol. 12 (1943) pp. 197-213.

4. I. Kaplansky, Projections in Banach algebras, Ann. of Math. vol. 53 (1951) pp. 235-249.

5. F. J. Murray and J. von Neumann, Rings of operators, Ann. of Math. vol. 37 (1936) pp. 116-229.

6. - Rings of operators. II, Trans. Amer. Math. Soc. vol. 41 (1937) pp. 208-248.

7. J. von Neumann, On rings of operators. Reduction theory, Ann. of Math. vol. 50 (1949) pp. 401-485.

InStitute for Advanced Study, Princeton, N. J. 